\title{
Education of medical problems in pregnancy - a survey of general internal medicine trainees in the West Midlands
}

\author{
Author: Paarul Prinja
}

Aims

To survey medical registrars within the West Midlands to ascertain the training that they have received on how to deal with medical problems in pregnancy and their confidence in doing so.

\section{Methods}

A questionnaire (via SurveyMonkey) was sent to all general internal medicine (GIM) trainees within the West Midlands in April 2017. Questions aimed to identify how frequently medical registrars are called to see a pregnant patient and the supervision that they received. The questionnaire also aimed to identify the forms of training available, the confidence of trainees in dealing with key presentations outlined in the curriculum and suggestions for further training.

\section{Results}

One-hundred and fourteen trainees responded; $91 \%$ of trainees intended to accredit with GIM. Despite being a competency within the GIM curriculum, $45 \%$ of trainees had not received any formal training on how to deal with medical problems in pregnancy.

$42 \%$ of trainees are seeing women who have medical problems in pregnancy at least every 2 months. Only 15\% of trainees received direct feedback or supervision regarding their management of these women.

While the majority of trainees (85\%) felt confident in managing venous thromboembolism in pregnancy, only $17 \%$ felt confident prescribing medication in pregnancy. Trainees felt more confident at managing sepsis in pregnancy than epilepsy or asthma ( $42 \%$ vs $28 \%$ vs $12 \%$ )

The majority (56\%) of those who had received training had done so through lectures. $92 \%$ of trainees feel that they would benefit from further training on how to deal with medical problems in pregnancy. $42 \%$ of trainees feel that they would benefit from simulation-based training; $33 \%$ of those surveyed would like a placement within an obstetric medicine department. $20 \%$ would like workshop or small group teaching, with the rest favouring lectures.

\section{Conclusions}

Despite being a competency for GIM, training and confidence of medical registrars on how to deal with medical problems in pregnancy in the West Midlands is lacking. The development of a maternal medicine simulation day for medical registrars, as well as case-based teaching at training days, should help to address this. Going forward, the development of obstetric medicine as a medical specialty will allow for more training to be delivered to doctors across various centres in the UK. Locally, more feedback and supervision should be given to trainees who see these patients. This requires supervising consultants to also be confident and competent at managing medical problems in pregnancy. This is an area for further work.

\section{Conflict of interest statement}

None declared. 